\title{
THE
}

1995

\section{Quantum integrability and action operators in spin dynamics}

\author{
Stefan Weigert \\ Gerhard Müller \\ University of Rhode Island, gmuller@uri.edu
}

Follow this and additional works at: https://digitalcommons.uri.edu/phys_facpubs

Terms of Use

All rights reserved under copyright.

\section{Citation/Publisher Attribution}

Stefan Weigert and Gerhard Müller. Quantum integrability and action operators in spin dynamics. Chaos, Solitons, and Fractals 5 (1995), 1419-1438.

Available at http://www.sciencedirect.com/science/article/pii/096007799500021U.

This Article is brought to you for free and open access by the Physics at DigitalCommons@URI. It has been accepted for inclusion in Physics Faculty Publications by an authorized administrator of DigitalCommons@URI. For more information, please contact digitalcommons-group@uri.edu. 


\title{
Quantum integrability and action operators in spin dynamics
}

\author{
Stefan Weigert ${ }^{1}$ and Gerhard Müller ${ }^{2}$ \\ ${ }^{1}$ Institut für Physik, Universität Basel, CH-4056 Basel, Switzerland. \\ ${ }^{2}$ Department of Physics, University of Rhode Island, Kingston RI 02881-0814, USA.
}

\begin{abstract}
A new formulation of the quantum integrability condition for spin systems is proposed. It eliminates the ambiguities inherent in formulations derived from a direct transcription of the classical integrability criterion. In the new formulation, quantum integrability of an $N$-spin system depends on the existence of a unitary transformation which expresses the Hamiltonian as a function of $N$ action operators. All operators are understood to be algebraic expressions of the spin-components with no restriction to any finite-dimensional matrix representation. The consequences of quantum (non-)integrability on the structure of quantum invariants are discussed in comparison with the consequences of classical (non-)integrability on the corresponding classical invariants. Our results indicate that quantum integrability is universal for systems with $N=1$ and contingent for systems with $N \geq 2$.
\end{abstract}

\section{INTRODUCTION}

In the early days of analytical dynamics, integrability was understood to be primarily a question of the development and application of adequate mathematical tools. Gradually it became clear that it is, in fact, an intrinsic property which some dynamical systems possess and others lack. The emerging awareness that seemingly simple dynamical systems will forever elude an analytical solution was painful to researchers at the time, only partly alleviated by their accomplishment of having found a general integrability condition for Hamiltonian systems. This criterion made it possible to identify integrable systems by means which amount to less than an explicit solution. [1]

In today's world of high-speed computers, the question of analytical solvability has lost much of its importance, but the integrability condition as a discriminant between two classes of systems with qualitatively distinct dynamical properties has remained at the core of research in nonlinear dynamics. The far-reaching implications of integrability and the dramatic consequences of nonintegrability have been elucidated in great detail by a combination of analytical and numerical methods. [2]

For any given (integrable or nonintegrable) classical Hamiltonian system, a family of quantum-mechanical systems with corresponding dynamical variables can be constructed by well-established procedures based on the correspondence principle. This raises the question as to what extent the classical attribute of integrability or the lack of it is still reflected in the properties of the quantized system.

Quantum chaos research has revealed a wealth of phenomena which distinguish quantized nonintegrable systems from quantized integrable systems. [3-6] Are the signatures of quantum chaos quantum manifestations of classical nonintegrability or manifestations of quantum nonintegrability? The second alternative implies the existence of a quantum integrability condition which can stand on its own, i.e. which is not merely a classical label attached to a quantized system.
The classical integrability condition for a system with $N$ degrees of freedom requires the existence of $N$ functionally independent analytic invariants in involution. When this condition is translated offhandedly into the requirement that there exist $N$ independent commuting operators, then it fails to state two important qualifications: (i) Which operators should be counted? (ii) What makes two operators independent? Various attempts at clarifying these points can be found in the literature, [79] but none seems to be watertight. [10]

In this paper we present an alternative formulation of the quantum integrability condition, which avoids known loopholes and ambiguities. It does not lean on any classical concept, yet its predictions appear to be consistent with the classical criterion in the sense that quantized integrable systems are quantum integrable and quantized nonintegrable ones quantum nonintegrable. The notion of quantum nonintegrability as proposed here is not linked to algorithmic complexity.[11] Its effects are nevertheless compelling, as we shall see.

In order to achieve a simple and unified conceptualization and notation, we focus the entire discussion on (classical or quantum) spin systems. However, a transcription of the essential features to systems involving other types of degrees of freedom seems straightforward.

In Sec. II we paint the classical backdrop for the new quantum integrability concept. The classical integrability condition is reformulated in a way that provides a more direct link to its newly proposed quantum counterpart. We describe and employ a computational procedure for the construction of classical invariants, whose properties depend sensitively on whether the underlying Hamiltonian is integrable or not. $[12,13]$ In Sec. III we propose the existence of a unitary transformation which converts any quantum one-spin system into a function of a single quantum action - an operator with specific spectral properties. Such an expression defines quantum integrability and implies that the time evolution of any non-stationary operator can be determined explicitly. We employ quantum invariants, constructed by a procedure analogous to the one used previously for classical invariants, [13] to produce analytical and numerical evidence in 
support of the proposition that all one-spin systems are quantum integrable. In Sec. IV we demonstrate by the same methods that the spectral consequences of quantum integrability are not common to all two-spin systems, only to some. The implication is that a unitary transformation expressing the quantum two-spin Hamiltonian as a function of two action operators exists only in some cases. In the concluding Sec. V, we outline possibilities to further corroborate the findings presented here by alternative methods based on perturbation expansions.

\section{INTEGRABLE AND NONINTEGRABLE CLASSICAL SPIN SYSTEMS}

Consider a system of $N$ classical spins length $S$,

$$
\begin{aligned}
\mathbf{S}_{n}= & \left(S_{n, x}, S_{n, y}, S_{n, z}\right) \\
= & S\left(\sin \vartheta_{n} \cos \varphi_{n}, \sin \vartheta_{n} \sin \varphi_{n}, \cos \vartheta_{n}\right), \\
& n=1, \ldots, N .
\end{aligned}
$$

The classical spin length is an invariant of the motion. The kinetics on the product of $N$ spheres with $S=$ const is governed by the Poisson brackets between spin components,

$$
\left\{S_{n, \alpha}, S_{n^{\prime}, \beta}\right\}=-\delta_{n n^{\prime}} \sum_{\gamma=x, y, z} \varepsilon_{\alpha \beta \gamma} S_{n, \gamma} .
$$

Each spin contributes one degree of freedom to the system and can be described in terms of one pair of canonical coordinates,

$$
p_{n}=S \cos \vartheta_{n}, \quad q_{n}=\varphi_{n} .
$$

The associated $2 N$-dimensional phase space is bounded. [14] The value of the constant $S$ is irrelevant for the issues discussed here. Hence it will be suppressed in any list of independent variables.

The algebra of dynamical variables for a system of classical spins consists of all analytic (piecewise smooth) phase-space functions. Any such function can be regarded as the Hamiltonian

$$
H=H\left(\mathbf{S}_{1}, \ldots, \mathbf{S}_{N}\right)
$$

of a dynamical system. In that role it determines the time evolution of any other dynamical variable $F\left(\mathbf{S}_{1}, \ldots, \mathbf{S}_{N}\right)$ according to Hamilton's equation of motion,

$$
\frac{d F}{d t}=\{H, F\} \text {. }
$$

For a specific Hamiltonian $H$, there may exist phasespace functions $I\left(\mathbf{S}_{1}, \ldots, \mathbf{S}_{N}\right)$ with the property

$$
\frac{d I}{d t}=\{H, I\}=0 .
$$

These are analytic invariants or integrals of the motion. Analyticity guarantees that $\{H, I\}$ is well-defined; invariance $(d I / d t=0)$ then follows from $\{H, I\}=0$. Nonanalytic invariants are functions $I\left(\mathbf{S}_{1}, \ldots, \mathbf{S}_{N}\right)$ which satisfy $d I / d t=0$, but for which $\{H, I\}$ cannot be evaluated due to lack of smoothness. $[12,13]$

The phase flow generated by a given Hamiltonian must belong to one of two distinct types. (i) Regular flow: The entire $2 N$-dimensional phase space is foliated into $N$-dimensional tori; individual phase points wind around these tori periodically or quasi-periodically. (ii) Irregular flow: A fraction of phase points are not confined to $N$ dimensional manifolds; their flow is chaotic. Important for our study is that this clear-cut phenomenological classification of flows is rigorously related to an integrability criterion for the flow-generating Hamiltonians.

\section{A. Classical integrability condition}

A classical system with $N$ degrees of freedom, specified by some Hamiltonian (4), is integrable if three conditions are fulfilled. [15]

C1: There exist $N$ analytic invariants $I_{n}\left(\mathbf{S}_{1}, \ldots, \mathbf{S}_{N}\right)$ : $\left\{H, I_{n}\right\}=0, n=1, \ldots, N$

C2: All pairs of these invariants are in involution: $\left\{I_{n}, I_{n^{\prime}}\right\}=0$.

C3: The invariants $I_{n}$ are functionally independent, i.e. the directions $\nabla I_{n}$ are linearly independent almost everywhere in phase space.

Otherwise the system is nonintegrable. It is impossible to have more than $N$ analytic invariants which are functionally independent and in involution. Therefore, $H$ is either equal to one of the $N$ invariants $I_{n}$, or else it can be written as a smooth function $H=\tilde{H}\left(I_{1}, \ldots, I_{N}\right)$. A set of $N$ invariants $I_{n}$ satisfying conditions C2 and C3 determine exactly one torus structure in phase space. Any smooth function $\tilde{H}\left(I_{1}, \ldots, I_{N}\right)$ specifies a particular flow on that same torus structure.

The action variables $J_{n}\left(\mathbf{S}_{1}, \ldots, \mathbf{S}_{N}\right)$ are a special set of invariants. They represent $N$ canonical momenta, for which the conditions $\mathrm{C} 2$ and $\mathrm{C} 3$ are necessarily satisfied. If the flow is generated by an action, all trajectories (except fixed points and separatrices) are closed and have the same period in time. The time evolution of any other dynamical variable $F\left(\mathbf{S}_{1}, \ldots, \mathbf{S}_{N}\right)$ for arbitrary initial conditions is then also periodic, but (generally) anharmonic. The associated intensity spectrum consists of a set of equidistant lines at integer frequencies.

The $N$ actions $J_{n}$ thus generate $N$ periodic flows in $N$ linearly independent directions on each torus. The flow of $H=\mathcal{H}\left(J_{1}, \ldots, J_{N}\right)$ can then be interpreted as a superposition of these $N$ fundamental periodic flows, determined by the linear time dependence of the angle variables, $\theta_{n}(t)=\omega_{n} t+\theta_{n}^{(0)}$; the characteristic frequencies $\omega_{n}=\partial \mathcal{H} / \partial J_{n}$ vary from one torus to another. The time evolution of a dynamical variable $F=$ $\mathcal{F}\left(J_{1}, \theta_{1}, \ldots, J_{N}, \theta_{N}\right)$ with initial conditions on a given torus is generally quasiperiodic. Its intensity spectrum 
consists of a superposition of lines at the sums and differences of the frequencies $\omega_{n}$. Without loss of generality, the classical integrability conditions $\mathrm{C} 1-\mathrm{C} 3$ can be replaced by a single requirement:

$\mathrm{CJ}(N)$ : A given Hamiltonian, $H\left(\mathbf{S}_{1}, \ldots, \mathbf{S}_{N}\right)$, is integrable if there exists a canonical transformation which converts it into a smooth function of $N$ actions $J_{n}$ : $H=\mathcal{H}\left(J_{1}, \ldots, J_{N}\right)$. The actions are a set of invariant canonical momenta, and each $J_{n}$ generates a phase flow whose spectrum consists of uniformly spaced lines.

This alternative integrability criterion is rarely used in classical mechanics because the additional requirement that the $N$ invariants be canonical momenta would make any proof of integrability unnecessarily difficult. However, it is $\mathrm{CJ}(N)$ and not $\mathrm{C} 1-\mathrm{C} 3$ that can be translated most directly into a meaningful quantum integrability condition as we shall see. As a preparation to our main theme (Secs. III and IV), we need to discuss a general method for constructing invariants in classical systems - a method which can also be adapted to quantum mechanics.

\section{B. One degree of freedom}

The Hamiltonian $H(\mathbf{S})$ of a one-spin system is an analytic invariant, the only one required to render the system integrable. Further smooth invariants are necessarily functionally dependent on $H$. Invariants can be constructed as the time average of an arbitrary dynamical variable $F(\mathbf{S})$ over individual phase-space trajectories,

$$
I_{F}(\mathbf{S}) \equiv\langle F\rangle=\lim _{T \rightarrow \infty} \frac{1}{T} \int_{0}^{T} d t F(\mathbf{S}(t))=\frac{1}{\tau} \int_{0}^{\tau} d t F(\mathbf{S}(t)),
$$

where the variable $\mathbf{S}$ on the left denotes the initial condition of the path $\mathbf{S}(t)$ in the integral on the right. The third equality uses the fact that in one-spin systems the time evolution is periodic on all trajectories except fixed points and separatrices. The phase-space function $I_{F}$ is an invariant by construction. This is an analytic invariant because the torus structure guarantees a smooth dependence of the integral on the initial condition $\mathbf{S}$, and it is functionally dependent on $H$ because both $I_{F}$ and $H$ assume a unique value for all initial conditions on a given torus: $I_{F}=\tilde{I}_{F}(H)$ or $H=\tilde{H}\left(I_{F}\right)$. For the special dynamical variable $F=p \dot{q}$ with $p, q$ from (3), the integral ( 7 ) yields the action

$$
J \equiv \frac{1}{2 \pi} \oint p d q=\frac{\tau}{2 \pi}\langle p \dot{q}\rangle
$$

again as a function of the energy. Conversely, we have $H=\mathcal{H}(J)$ and, by implication, $I_{F}=\mathcal{I}_{F}(J)$.

\section{Two degrees of freedom}

The integrability of a two-spin system specified by some energy function $H\left(\mathbf{S}_{1}, \mathbf{S}_{2}\right)$ hinges on the existence of a second independent analytic invariant $I\left(\mathbf{S}_{1}, \mathbf{S}_{2}\right)$. For an integrable system, this second invariant is not unique, and no general prescription for obtaining an explicit expression for $I$ is known. However, a general method exists for the evaluation of that invariant on a dense set of phase points with full measure. This method was proposed earlier, $[12,13]$ and its usefulness for practical applications was demonstrated by numerical implementations.

The method extends the prescription given in (7). Choose an arbitrary dynamical variable $F\left(\mathbf{S}_{1}, \mathbf{S}_{2}\right)$ and determine its time average over phase-space trajectories as a function of the initial conditions:

$$
I_{F}\left(\mathbf{S}_{1}, \mathbf{S}_{2}\right) \equiv\langle F\rangle=\lim _{T \rightarrow \infty} \frac{1}{T} \int_{0}^{T} d t F\left(\mathbf{S}_{1}(t), \mathbf{S}_{2}(t)\right) .
$$

The four-dimensional phase space is foliated by twodimensional invariant tori. Therefore, the invariant $\langle F\rangle$ represents a function of only two variables - the two action coordinates $J_{1}$ and $J_{2}$. They uniquely specify the torus which contains the phase point $\left(\mathbf{S}_{1}, \mathbf{S}_{2}\right)$. The function

$$
\mathcal{I}_{F}\left(J_{1}, J_{2}\right)=I_{F}\left(\mathbf{S}_{1}, \mathbf{S}_{2}\right)
$$

is an analytic invariant. Hence, it satisfies condition C1, but it may or may not satisfy condition $\mathrm{C} 3$, while property $\mathrm{C} 2$ is redundant for $N=2$. The functional relationship between the invariant $I_{F}$ and the Hamiltonian $H$ can be identified in a plot of $I_{F}$ versus $H$. If $I_{F}$ is functionally dependent on $H$, the graph is a piecewise smooth line. Otherwise the points $\left(I_{F}, H\right)$, each representing an individual torus in phase space, fill a two-dimensional region.

The existence of a smooth and functionally independent second invariant, which is guaranteed by integrability, can be visualized by a previously developed construction.[13] Determine, via time average (9), two analytic invariants $I_{F}=\mathcal{I}_{F}\left(J_{1}, J_{2}\right)$ and $I_{G}=\mathcal{I}_{G}\left(J_{1}, J_{2}\right)$ which are functionally independent of $H=\mathcal{H}\left(J_{1}, J_{2}\right)$ and functionally independent of each other. The dependence of these invariants on the actions $J_{1}, J_{2}$ is, in general, not known explicitly, but for individual tori their numerical values can be determined everywhere in phase space. The points $\left(H, I_{F}, I_{G}\right)$ then form a piecewise smooth invariant-surface in a three-dimensional diagram. It is an image of the $\left(J_{1}, J_{2}\right)$-plane in $\left(H, I_{F}, I_{G}\right)$-space. The function

$$
H=\tilde{H}\left(I_{F}, I_{G}\right)
$$

is piecewise smooth. Each invariant torus in phase space determines a point on the invariant-surface and a point in the plane of actions. 
In the phase flow of a nonintegrable two-spin system $H\left(\mathbf{S}_{1}, \mathbf{S}_{2}\right)$, a fraction of the invariant tori are destroyed. The remaining ones are no longer dense anywhere, but they still occupy a volume with nonzero measure. The chaotic regions between intact tori exhaust the remaining measure. Nonintegrability causes dramatic changes in the structure of invariant-surfaces. Suppose that two invariants $I_{F}$ and $I_{G}$ have been determined which are functionally independent of the Hamiltonian $H$ and of each other. The points $\left(H, I_{F}, I_{G}\right)$ resulting from all invariant structures in phase space (tori, cantori, chaotic regions, periodic orbits) then form a strange invariant-surface, an almost two-dimensional object in three-dimensional space, pieces of which may look like a smooth surface on a large scale. The function $H=\tilde{H}\left(I_{F}, I_{G}\right)$ is nowhere continuous. The differences between smooth and strange invariant-surfaces will be illustrated in Sec. IV and compared with the properties of corresponding quantummechanical structures.

\section{QUANTUM SPIN SYSTEMS: ONE DEGREE OF FREEDOM}

A quantum mechanical spin is described by a 3component vector operator $\widehat{\mathbf{S}}$. Its components satisfy the familiar commutation relations

$$
\left[\widehat{S}_{\alpha}, \widehat{S}_{\beta}\right]=i \hbar \sum_{\gamma=x y z} \varepsilon_{\alpha \beta \gamma} \widehat{S}_{\gamma}
$$

The operator

$$
\widehat{\mathbf{S}}^{2}=\widehat{S}_{x}^{2}+\widehat{S}_{y}^{2}+\widehat{S}_{z}^{2},
$$

which represents the square of the quantum spin length, commutes with any Hamiltonian of the form

$$
\widehat{H}=H(\widehat{\mathbf{S}}) .
$$

or with any dynamical variable of this system as expressed by a (generally non-stationary) Hermitian operator

$$
\widehat{F}=F(\widehat{\mathbf{S}})
$$

The representations of the spin algebra (12) can be labeled by the eigenvalues $\hbar^{2} s(s+1), s=1 / 2,1,3 / 2, \ldots$ of the operator $\widehat{\mathbf{S}}^{2}$. For each value of $s$ there is an irreducible $(2 s+1)$-dimensional matrix representation $\Gamma_{s}$ of the group $S U(2)$.

In the context of this study, any function of the spin components such as $H(\widehat{\mathbf{S}})$ or $F(\widehat{\mathbf{S}})$ is defined as an operator on the full Hilbert space. This is equivalent to considering all subspaces $\Gamma_{s}$ simultaneously by expressing the operators as infinite block matrices. A superscript $s$ such as in $\widehat{F}^{s}=F^{s}(\widehat{\mathbf{S}})$ will mark any $(2 s+1)$-dimensional matrix representation of an operator. Operators acting in the full Hilbert space are representation-independent and will be called algebraic.

\section{A. Unitary transformation and action operator}

Any unitary transformation $\widehat{U}=U(\widehat{\mathbf{S}})$ applied to a spin operator $\widehat{\mathbf{S}}$ produces again a spin operator,

$$
\widehat{\mathbf{S}}^{\prime}=\widehat{U} \widehat{\mathbf{S}} \widehat{U}^{\dagger}=\mathbf{S}^{\prime}(\widehat{\mathbf{S}}),
$$

i.e. it leaves the structure of the quantum spin algebra (12) unchanged:

$$
\left[\widehat{S}_{\alpha}^{\prime}, \widehat{S}_{\beta}^{\prime}\right]=i \hbar \sum_{\gamma=x y z} \varepsilon_{\alpha \beta \gamma} \widehat{S}_{\gamma}^{\prime} .
$$

The rotations in spin space are a subset of these transformations with a linear dependence on the spin components. Under $U(\widehat{\mathbf{S}})$, the Hamiltonian (14) and the dynamical variable (15) are transformed into algebraic expressions of the new spin vector: $H(\widehat{\mathbf{S}})=H^{\prime}\left(\widehat{\mathbf{S}}^{\prime}\right)$ and $F(\widehat{\mathbf{S}})=F^{\prime}\left(\widehat{\mathbf{S}}^{\prime}\right)$, respectively.

Suppose there exists a special unitary transformation, $\widehat{\mathcal{U}}=\mathcal{U}(\widehat{\mathbf{S}})$ with

$$
\widehat{\mathbf{J}}=\widehat{\mathcal{U}} \widehat{\mathbf{S}} \widehat{\mathcal{U}}^{\dagger}=\mathbf{J}(\widehat{\mathbf{S}})
$$

which converts a given one-spin Hamiltonian $H(\widehat{\mathbf{S}})$ into a function of a single component of the transformed spin:

$$
H(\widehat{\mathbf{S}})=\mathcal{H}\left(\hat{J}_{z}\right) .
$$

What are the consequences of such a transformation? The operator $\hat{J}_{z}$ is appropriately named action operator. Its eigenvalue spectrum in each subspace $\Gamma_{s}$ consists of $2 s+1$ uniformly spaced levels,

$$
\hat{J}_{z}^{s}=\sum_{m=-s}^{+s}|m, s\rangle m \hbar\langle m, s| .
$$

The operator $\hat{J}_{z}$ is an invariant, $\left[\widehat{H}, \hat{J}_{z}\right]=0$, and it shares many properties with the classical action variable as will be discussed. When the same transformation which produces (19) is applied to a (non-stationary) dynamical variable $F(\widehat{\mathbf{S}})$, the resulting expression,

$$
F(\widehat{\mathbf{S}})=\mathcal{F}(\hat{\mathbf{J}})
$$

depends not only on the quantum action $\hat{J}_{z}$ but also on the non-stationary components $\hat{J}_{x}, \hat{J}_{y}$. We shall see that these two operators assume a role similar to that of a classical angle variable. On the basis of these properties we propose the following quantum integrability condition:

QJ(1): A given one-spin Hamiltonian $\widehat{H}=H(\widehat{\mathbf{S}})$ is quantum integrable if there exists a unitary transformation $\mathcal{U}(\widehat{\mathbf{S}})$ which converts it into a function of the action operator $\hat{J}_{z}$. The action operator is a quantum invariant whose spectrum in any subspace $\Gamma_{s}$ consists of a set of $2 s+1$ uniformly spaced levels. 
The classical integrability of all one-spin Hamiltonians $H(\widehat{\mathbf{S}})$ is an elementary result. The criterion CJ(1) is satisfied universally. The canonical transformation to action-angle coordinates leading to the expression $H(\mathbf{S})=\mathcal{H}(J)$ for the Hamiltonian, and to $F(\mathbf{S})=$ $\mathcal{F}(J, \theta)$ for any dynamical variable of interest, can be established by constructive methods.[2] Correspondingly, we assert that all one-spin Hamiltonians $H(\widehat{\mathbf{S}})$ are quantum integrable, i.e. that the criterion QJ(1) is satisfied universally. However, we have yet to design a constructive method which leads to the expressions $\mathcal{H}\left(\hat{J}_{z}\right)$ and $\mathcal{F}(\hat{\mathbf{J}})$ for the Hamiltonian and a non-stationary operator, respectively.

In the classical canonical transformation to actionangle coordinates, the transformed Hamiltonian $\mathcal{H}(J)$ may have an additional dependence on the (constant) classical spin length $S$. Likewise, the algebraic expression $\mathcal{H}\left(\hat{J}_{z}\right)$ resulting from the special unitary transformation $\mathcal{U}(\widehat{\mathbf{S}})$ may contain an explicit dependence on the operator $\widehat{\mathbf{S}}^{2}=\hat{\mathbf{J}}^{2}$, which represents the (invariant) quantum spin length. Since this invariant commutes with all operators $F(\widehat{\mathbf{S}})$, it has no bearing on the question of quantum integrability. It will therefore be suppressed in any list of independent variables, as was the classical spin length.

\section{B. Quantum invariants}

In the absence of a general recipe for determining the relation (19) between Hamiltonian and quantum action in one-spin systems, we provide various kinds of indirect evidence which support the postulate of universal quantum integrability in these systems, and we show that the consequences of quantum integrability have much in common with those of classical integrability. Part of this evidence can be inferred from relations between invariants as obtained via time average of dynamical variables, analogously to the classical construction presented in Sec. II.

Consider a Hamiltonian $H(\widehat{\mathbf{S}})$ and a dynamical variable $F(\widehat{\mathbf{S}})$. The solution of the Heisenberg equation of motion,

$$
\frac{d \widehat{\mathbf{S}}}{d t}=\frac{i}{\hbar}[\widehat{H}, \widehat{\mathbf{S}}]
$$

is a one-parameter family of operators, $\widehat{\mathbf{S}}(t)$, and the time average of the operator $F(\widehat{\mathbf{S}}(t))$ defines an operator,

$$
\hat{I}_{F}=\langle\widehat{F}\rangle \equiv \lim _{T \rightarrow \infty} \frac{1}{T} \int_{0}^{T} d t F(\widehat{\mathbf{S}}(t))
$$

which is an invariant by construction. In the subspace of $\Gamma_{s}$, the dynamical variable $\widehat{F}$ is given by a matrix $\widehat{F}^{s}$ with elements

$$
F_{m m^{\prime}}^{s}(t)=\left\langle m, s\left|\widehat{F}^{s}\right| m^{\prime}, s\right\rangle \exp \left[i\left(E_{m}^{s}-E_{m^{\prime}}^{s}\right) t / \hbar\right],
$$

in the energy representation

$$
\widehat{H}^{s}|m, s\rangle=E_{m}^{s}|m, s\rangle .
$$

Taking the time average of $\widehat{F}^{s}$ then amounts to setting all its off-diagonal matrix elements equal to zero. If degenerate energy levels occur, the eigenvectors $|m, s\rangle$ can be chosen in such a way that no time-independent offdiagonal matrix elements remain.

If our postulate is correct, then any quantum invariant obtained from the time average of a dynamical variable must also be expressible as a function of the quantum action $\hat{J}_{z}$. In systems where the Hamiltonian is already given as a function of $\hat{J}_{z}$, the time average (23) can be carried out analytically, producing invariants in the form of explicit functions of $\hat{J}_{z}$.

\section{Dynamics of $\widehat{H}=H\left(\widehat{S}_{z}\right)$ : exact calculation}

Consider a quantum spin in a constant magnetic field pointing in $z$-direction. With $\widehat{\mathbf{S}} \equiv \hat{\mathbf{J}}$, i.e. $\widehat{\mathcal{U}}=1$, the Hamiltonian is a linear function of the action:

$$
\widehat{H}=-\frac{g e}{2 m c} B \widehat{S}_{z} \equiv-\omega_{B} \hat{J}_{z}=\mathcal{H}\left(\hat{J}_{z}\right)
$$

The equations of motion (22) for the components of $\hat{\mathbf{J}}$ or the associated ladder operators $\hat{J}_{ \pm}=\hat{J}_{x} \pm i \hat{J}_{y}$ are then readily solved algebraically:

$$
\begin{aligned}
& \hat{J}_{ \pm}(t)=\hat{J}_{ \pm}(0) \exp \left[ \pm i \omega_{B} t / \hbar\right] \\
& \hat{J}_{z}(t)=\hat{J}_{z}(0)
\end{aligned}
$$

The harmonic time dependence of the operators $\hat{J_{ \pm}}$indicate their kinship to a classical angle variable. The time evolution of an arbitrary dynamical variable $\widehat{F}=F(\widehat{\mathbf{S}})=$ $\mathcal{F}(\hat{\mathbf{J}})$ (here with $F=\mathcal{F}$ ) is then obtained by insertion of (27). As in classical mechanics (Sec. II), the dynamics generated by an action is universally periodic. Given the explicit time-dependence of $\mathcal{F}(\hat{\mathbf{J}})$, we can evaluate its time average. For a simple illustration, consider the dynamical variable

$$
\widehat{F}=\hat{J}_{x}^{2} \hat{J}_{z}+\hat{J}_{z} \hat{J}_{x}^{2}
$$

rewritten in the form

$$
\widehat{F}=\left(\hat{\mathbf{J}}^{2}-\hat{J}_{z}^{2}\right) \hat{J}_{z}+\frac{1}{4}\left[\left(\hat{J}_{+}^{2}+\hat{J}_{-}^{2}\right) \hat{J}_{z}+\hat{J}_{z}\left(\hat{J}_{+}^{2}+\hat{J}_{-}^{2}\right)\right]
$$

for easy insertion of the solutions (27). The time average leaves the first term intact and wipes out the second one. The resulting invariant,

$$
\hat{I}_{F}=\left(\hat{\mathbf{J}}^{2}-\hat{J}_{z}^{2}\right) \hat{J}_{z}=\mathcal{I}_{F}\left(\hat{J}_{z}\right)
$$

is indeed a function of the action operator as we claim it must necessarily be. 
This result holds for arbitrary operators $\widehat{F}$ expressible as power series in $\hat{J}_{x}, \hat{J}_{y}, \hat{J}_{z}$. For example, the $n^{t h}$ power of $\hat{J}_{x}$ has the form

$$
\hat{J}_{x}^{n}(t)=\frac{1}{2^{n}} \sum_{k=0}^{n} P_{k}^{n}\left(\hat{J}_{+}, \hat{J}_{-}\right) \exp \left[-i(n-2 k) \omega_{B} t / \hbar\right],
$$

where $P_{k}^{n}\left(\hat{J}_{+}, \hat{J}_{-}\right)$is a polynomial of order $n$ containing all possible orderings of $k$ powers of $\hat{J}_{-}$and $(n-k)$ powers of $\hat{J}_{+}$. The time average vanishes unless $n$ is even, and then the only surviving term is the one with $k=n / 2$ :

$$
\left\langle\hat{J}_{x}^{n}(t)\right\rangle=\frac{1}{2^{n}} P_{n / 2}^{n}\left(\hat{J}_{+}, \hat{J}_{-}\right), \quad n \text { even }
$$

which is a sum of monomials $\widehat{\mathcal{Q}}_{n} \equiv \prod \hat{J}_{+}^{a_{1}} \hat{J}_{-}^{b_{1}} \hat{J}_{+}^{a_{2}} \hat{J}_{-}^{b_{2}} \ldots$, with $a=a_{1}+a_{2}+\ldots=n / 2$ and $b=b_{1}+b_{2}+\ldots=n / 2$. By virtue of the relations

$$
\hat{J}_{ \pm} \hat{J}_{\mp}=\hat{\mathbf{J}}^{2}-\hat{J}_{z}^{2} \pm \hbar \hat{J}_{z}, \quad \hat{J}_{z} \hat{J}_{ \pm}=\hat{J}_{ \pm} \hat{J}_{z} \pm \hbar \hat{J}_{ \pm},
$$

each term $\widehat{\mathcal{Q}}_{n}$ is a function of the quantum action $\hat{J}_{z}$. We can use the same argument to determine $\left\langle\hat{J}_{y}^{n}(t)\right\rangle$ and, more generally, time averages of polynomials and power series of $\hat{J}_{x}, \hat{J}_{y}, \hat{J}_{z}$. The final result is the time average of the non-stationary operator $\widehat{F}$ expressed as an explicit and unique function of the action operator:

$$
\widehat{I}_{F}=\langle\mathcal{F}(\hat{\mathbf{J}})\rangle=\mathcal{I}_{F}\left(\hat{J}_{z}\right)
$$

Turning the argument around, we conclude that if the operator $\widehat{F}=\mathcal{F}(\hat{\mathbf{J}})$ happens to be an invariant, then it must be expressible as a function of $\hat{J}_{z}$. All this reflects the existence of some algebraic dependence between any two invariants. It corresponds to the functional dependence between any two analytic invariants of a classical one-spin model (Sec. II.B).

Suppose the quantum one-spin Hamiltonian is given as a nonlinear function of a single spin component. A rotation in spin space turns that component into the quantum action:

$$
\widehat{H}=\mathcal{H}\left(\hat{J}_{z}\right)
$$

In this case, the equation of motion has explicit solutions of the form

$$
\begin{aligned}
\hat{J}_{ \pm}(t) & =\exp \left[i \mathcal{H}\left(\hat{J}_{z}\right) t / \hbar\right] \hat{J}_{ \pm}(0) \exp \left[-i \mathcal{H}\left(\hat{J}_{z}\right) t / \hbar\right] \\
\hat{J}_{z}(t) & =\hat{J}_{z}(0)
\end{aligned}
$$

The operators $\hat{J}_{ \pm}$still vary harmonically in time, but now the frequency is a function of the energy. This is also true for the angle variable of the corresponding classical system.

Given the solutions (36), it can still be shown that the time average of an arbitrary dynamical variable $\widehat{F}=$ $F(\widehat{\mathbf{S}})=\mathcal{F}(\hat{\mathbf{J}})$ becomes a function of $\hat{J}_{z}$ as in $(34)$, and if
$\widehat{F}$ is known as a polynomial or power series, then $\mathcal{I}_{F}\left(\hat{J}_{z}\right)$ can be determined as a polynomial or power series. Also, if any operator $F(\widehat{\mathbf{S}})$ is an invariant, it can b e reduced to the form $\mathcal{F}\left(\hat{J}_{z}\right)$. What remains to be shown is that an arbitrary one-spin Hamiltonian $H(\widehat{\mathbf{S}})$ can be brought into the form $\mathcal{H}\left(\hat{J}_{z}\right)$ by means of a unitary transformation. For that task we do not yet have a general prescription. However, the following results of a first-order perturbation calculation (Sec. III.D) and those of a numerical calculation (Sec. III.E) provide evidence that the underlying hypothesis is reasonable.

\section{Dynamics of $\widehat{H}=H_{0}\left(\widehat{S}_{z}\right)+\epsilon H_{1}(\widehat{\mathbf{S}})$ : perturbation calculation}

For a one-spin Hamiltonian of the form

$$
H(\widehat{\mathbf{S}})=H_{0}\left(\widehat{S}_{z}\right)+\epsilon H_{1}(\widehat{\mathbf{S}})
$$

with $\epsilon \ll 1$, the unitary transformation $\mathcal{U}(\widehat{\mathbf{S}})$ which turns $H(\widehat{\mathbf{S}})$ into a function of the action operator, $\mathcal{H}\left(\hat{J}_{z}\right)$, can be determined perturbatively. The prescripton for a firstorder calculation is simple and transparent. We apply the unitary transformation to the original Hamiltonian (37), expand it to leading order in $\epsilon$,

$$
\widehat{\mathcal{U}}^{\dagger} H(\widehat{\mathbf{S}}) \widehat{\mathcal{U}}=H(\widehat{\mathbf{S}})-\epsilon \frac{i}{\hbar}[u(\widehat{\mathbf{S}}), H(\widehat{\mathbf{S}})]+\mathcal{O}\left(\epsilon^{2}\right),
$$

and determine the operator $u(\widehat{\mathbf{S}})$ such that the offdiagonal parts of the commutator term and of the original Hamiltonian cancel each other. The remaining diagonal terms represent the Hamiltonian as a function of the action operator. Inserting $u(\widehat{\mathbf{S}})$ into the expansion of $(18)$,

$$
\widehat{\mathbf{J}}=\widehat{\mathcal{U}} \widehat{\mathbf{S}} \widehat{\mathcal{U}}^{\dagger}=\widehat{\mathbf{S}}+\epsilon \frac{i}{\hbar}[u(\widehat{\mathbf{S}}), \widehat{\mathbf{S}}]+\mathcal{O}\left(\epsilon^{2}\right),
$$

yields an operator $\hat{\mathbf{J}}$ whose components satisfy the spin commutation relations $(12)$ to $\mathcal{O}\left(\epsilon^{2}\right)$. For a simple illustration, consider the one-spin Hamiltonian,

$$
H(\widehat{\mathbf{S}})=\widehat{S}_{z}+\frac{\epsilon}{2} \widehat{S}_{x}^{2}=\widehat{S}_{z}+\frac{\epsilon}{4}\left(\widehat{\mathbf{S}}^{2}-\widehat{S}_{z}^{2}\right)+\frac{\epsilon}{8}\left(\widehat{S}_{+}^{2}+\widehat{S}_{-}^{2}\right)
$$

which we have rewritten, in the last equation, as the sum of a diagonal and an off-diagonal term. The operator $u(\widehat{\mathbf{S}})$ which diagonalizes $(38)$ to $\mathcal{O}(\epsilon)$ is found to have the form

$$
u(\widehat{\mathbf{S}})=\frac{i}{8}\left(\widehat{S}_{+}^{2}-\widehat{S}_{-}^{2}\right) .
$$

The resulting diagonal Hamiltonian reads

$$
\widehat{\mathcal{U}}^{\dagger} H(\widehat{\mathbf{S}}) \widehat{\mathcal{U}}=\widehat{S}_{z}+\frac{\epsilon}{4}\left(\widehat{\mathbf{S}}^{2}-\widehat{S}_{z}^{2}\right)
$$

or, in terms of the transformed spin $\widehat{\mathcal{U}} \widehat{\mathbf{S}} \widehat{\mathcal{U}}^{\dagger}=\hat{\mathbf{J}}$,

$$
\widehat{H}(\widehat{\mathbf{S}})=\mathcal{H}\left(\hat{J}_{z}\right)=\hat{J}_{z}+\frac{\epsilon}{4}\left(\hat{\mathbf{J}}^{2}-\hat{J}_{z}^{2}\right) .
$$


Note that the transformation generated by $u(\widehat{\mathbf{S}})$,

$$
\begin{aligned}
\hat{J}_{ \pm} & =\widehat{S}_{ \pm}+\frac{\epsilon}{4 \hbar}\left(\widehat{S}_{\mp} \widehat{S}_{z}+\widehat{S}_{z} \widehat{S}_{\mp}\right)+\mathcal{O}\left(\epsilon^{2}\right) \\
\hat{J}_{z} & =\widehat{S}_{z}-\frac{\epsilon}{\hbar}\left(\widehat{S}_{+}^{2}+\widehat{S}_{-}^{2}\right)+\mathcal{O}\left(\epsilon^{2}\right),
\end{aligned}
$$

is not just a rotation in spin space. Once the Hamiltonian has been brought into the form (43), we have reduced the problem to the one solved in Sec. III.C.

\section{E. Dynamics of $\widehat{H}=H(\widehat{\mathbf{S}})$ : numerical calculation}

For generic one-spin Hamiltonians such as (37) with unrestricted $\epsilon$, we can produce numerical results for quantum invariants. Their characteristic properties strongly support the existence of an action operator. We discuss the salient features in the context of a specific example. Consider the Hamiltonian

$$
\widehat{H}=\widehat{S}_{z}+\frac{1}{2} \widehat{S}_{x}^{2},
$$

and the invariant $\widehat{I}_{F}$ determined via time average from the dynamical variable

$$
\widehat{F}=\widehat{S}_{x}^{2} \widehat{S}_{z}+\widehat{S}_{z} \widehat{S}_{x}^{2}
$$

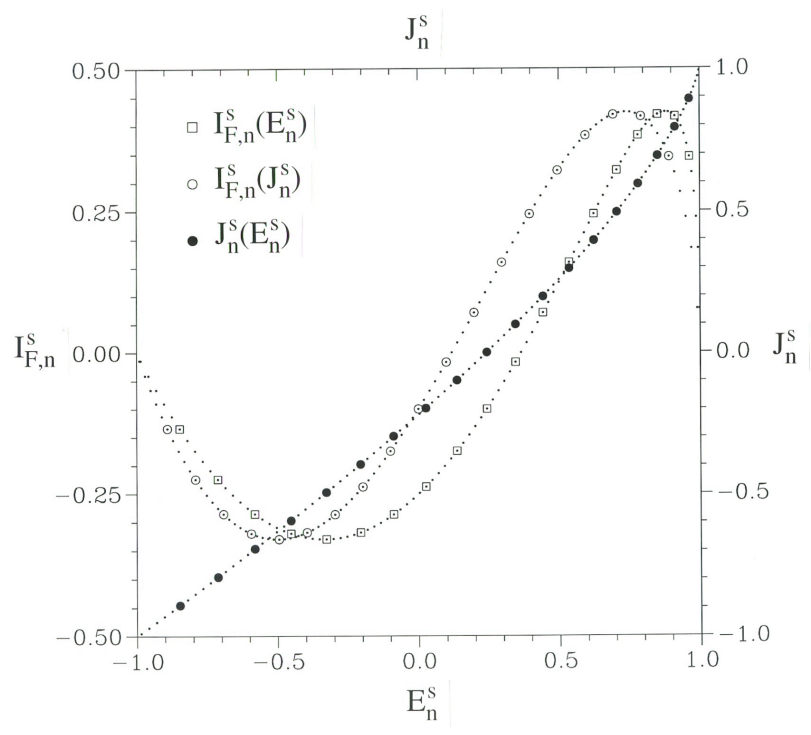

FIG. 1: The three quantum invariants $\widehat{H}=\widehat{S}_{z}+\widehat{S}_{x}^{2} / 2$ (energy), $\hat{J}_{z}$ (action), and $\widehat{I}_{F}=\langle\widehat{F}\rangle$ determined via time average from the non-stationary operator $\widehat{F}=\widehat{S}_{x}^{2} \widehat{S}_{z}+\widehat{S}_{z} \widehat{S}_{x}^{2}$, plotted one versus the other in the subspace $\Gamma_{s}$ with $s=50$ and with $\hbar=1 / \sqrt{s(s+1)}$. Each data set consists of $2 s+1=101$ points, of which every fifth is marked by a distinctive symbol (see legend).

In Fig. 1 we have compiled three sets of data for this model in the subspace of $\Gamma_{s}$ with $s=50$. The primary data obtained from the numerical calculation are the energy eigenvalues $E_{n}^{s}=\left\langle n, s\left|\widehat{H}^{s}\right| n, s\right\rangle$ and the values $I_{F, n}^{s}=\left\langle n, s\left|\widehat{F}^{s}\right| n, s\right\rangle$ of the invariant $\widehat{I}_{F}^{s}$. The set of points $\left(E_{n}^{s}, I_{F, n}^{s}\right)$, marked by open squares, exhibits two characteristic properties that reflect the universal integrability of quantum one-spin systems. (i) They fall onto a line which is smooth on a scale much larger than the average spacing between neighboring points. (ii) The sequence of successive spacings is smooth on the same large scale.

Property (i) suggests a natural sequential labeling, $J_{n}^{s} / \hbar=-s,-s+1, \ldots,+s$, of the points $\left(E_{n}^{s}, I_{F, n}^{s}\right)$ along that smooth line. These labels represent the eigenvalues of the action operator $\hat{J}_{z}$ in the subspace $\Gamma_{s}$. Property (ii) then implies that the two sets of points $\left(J_{n}^{s}, I_{F, n}^{s}\right)$ and $\left(E_{n}^{s}, J_{n}^{s}\right)$, marked by open and closed circles, respectively, also fall onto smooth lines. This is indeed the case. The smooth lines of points are strongly indicative of the algebraic dependence between any two of the three operators $\widehat{H}, \widehat{I}_{F}$, and $\widehat{J}_{z}$, i.e. indicative of the algebraic relations $\widehat{H}=\mathcal{H}\left(\widehat{J}_{z}\right)$ and $\widehat{I}_{F}=\mathcal{I}_{F}\left(\widehat{J}_{z}\right)$ which imply quantum integrability of this model system.

\section{QUANTUM SPIN SYSTEMS: TWO DEGREES OF FREEDOM}

Consider a two-spin Hamiltonian defined as an algebraic expression

$$
\widehat{H}=H\left(\widehat{\mathbf{S}}_{1}, \widehat{\mathbf{S}}_{2}\right) .
$$

The components of the two spins satisfy the commutation relations

$$
\left[\widehat{S}_{j \alpha}, \widehat{S}_{k \beta}\right]=i \hbar \delta_{j k} \sum_{\gamma=x y z} \varepsilon_{\alpha \beta \gamma} \widehat{S}_{j \gamma}, \quad j, k=1,2 .
$$

The operators, $\widehat{\mathbf{S}}_{1}^{2}$ and $\widehat{\mathbf{S}}_{2}^{2}$, which represent the lengths of the two spins, commute with each other, with the Hamiltonian (47), and with any dynamical variable,

$$
\widehat{F}=F\left(\widehat{\mathbf{S}}_{1}, \widehat{\mathbf{S}}_{2}\right) .
$$

With little loss of generality, we set $\widehat{\mathbf{S}}_{1}^{2}=\widehat{\mathbf{S}}_{2}^{2} \equiv \widehat{\mathbf{S}}^{2}$ and obtain, for each eigenvalue $\hbar^{2} s(s+1)$ of $\widehat{\mathbf{S}}^{2}$, a $(2 s+1)^{2}$ dimensional product representation $\Gamma_{s}=\Gamma_{1 s} \otimes \Gamma_{2 s}$ of the two-spin algebra (48). Any such representation of operators is again marked by a superscript $s$.

\section{A. Quantum integrability condition}

For the formulation of a quantum integrability condition applicable to two-spin systems, we consider unitary transformations $\widehat{U}=U\left(\widehat{\mathbf{S}}_{1}, \widehat{\mathbf{S}}_{2}\right)$ of the most general type. They transform the original spins $\widehat{\mathbf{S}}_{1}, \widehat{\mathbf{S}}_{2}$ into a new pair of spin operators,

$$
\widehat{\mathbf{S}}_{j}^{\prime}=\widehat{U} \widehat{\mathbf{S}}_{j} \widehat{U}^{\dagger}=\mathbf{S}_{j}^{\prime}\left(\widehat{\mathbf{S}}_{1}, \widehat{\mathbf{S}}_{2}\right), \quad j=1,2,
$$


i.e. into operators whose components satisfy the same basic commutation rules

$$
\left[\widehat{S}_{j \alpha}^{\prime}, \widehat{S}_{k \beta}^{\prime}\right]=i \hbar \delta_{j k} \sum_{\gamma=x y z} \varepsilon_{\alpha \beta \gamma} \widehat{S}_{j \gamma}^{\prime}, \quad j, k=1,2 .
$$

They convert the algebraically defined operators (47) and (49) into algebraic expressions of the new spin operators: $H\left(\widehat{\mathbf{S}}_{1}, \widehat{\mathbf{S}}_{2}\right)=H^{\prime}\left(\widehat{\mathbf{S}}_{1}^{\prime}, \widehat{\mathbf{S}}_{2}^{\prime}\right)$ and $F\left(\widehat{\mathbf{S}}_{1}, \widehat{\mathbf{S}}_{2}\right)=$ $F^{\prime}\left(\widehat{\mathbf{S}}_{1}^{\prime}, \widehat{\mathbf{S}}_{2}^{\prime}\right)$. Such transformations are not restricted to products $U_{1}\left(\widehat{\mathbf{S}}_{1}\right) U_{2}\left(\widehat{\mathbf{S}}_{2}\right)$ of single-spin transformations. In generalization of the criterion QJ(1) for one-spin systems as stated in Sec. III, we propose the following quantum integrability condition for two-spin systems:

QJ(2): A given two-spin Hamiltonian $\widehat{H}=H\left(\widehat{\mathbf{S}}_{1}, \widehat{\mathbf{S}}_{2}\right)$ is quantum integrable if there exists a unitary transformation $\mathcal{U}\left(\widehat{\mathbf{S}}_{1}, \widehat{\mathbf{S}}_{2}\right)$ which converts the spin operators $\widehat{\mathbf{S}}_{j}, j=1,2$, into new spin operators,

$$
\widehat{\mathbf{J}}_{k}=\widehat{\mathcal{U}} \widehat{\mathbf{S}}_{k} \widehat{\mathcal{U}}^{\dagger}=\mathbf{J}_{k}\left(\widehat{\mathbf{S}}_{1}, \widehat{\mathbf{S}}_{2}\right), \quad j=1,2,
$$

such that the Hamiltonian turns into a function of two action operators,

$$
H\left(\widehat{\mathbf{S}}_{1}, \widehat{\mathbf{S}}_{2}\right)=\mathcal{H}\left(\hat{J}_{1 z}, \hat{J}_{2 z}\right) .
$$

The eigenvalue spectra $\hat{J}_{1 z}, \hat{J}_{2 z}$ in each subspace $\Gamma_{s}$ consist of $2 s+1$ uniformly spaced levels:

$$
\begin{aligned}
& \hat{J}_{1, z}^{s}=\sum_{m, n=-s}^{+s}|m, n, s\rangle m \hbar\langle m, n, s|, \\
& \hat{J}_{2, z}^{s}=\sum_{m, n=-s}^{+s}|m, n, s\rangle n \hbar\langle m, n, s| .
\end{aligned}
$$

If a given Hamiltonian can be brought into this form, then the time evolution of the non-stationary spin components, $\hat{J}_{k x}, \hat{J}_{k y}, k=1,2$, can be stated explicitly and inserted into the transformed algebraic expression of any dynamical variable

$$
F\left(\widehat{\mathbf{S}}_{1}, \widehat{\mathbf{S}}_{2}\right)=\mathcal{F}\left(\widehat{\mathbf{J}}_{1}, \widehat{\mathbf{J}}_{2}\right) .
$$

It is straightforward to generalize QJ(2) to the integrability condition $\mathrm{QJ}(N)$ for an $N$-spin system. The quantum integrability condition $\mathrm{QJ}(N)$ is a more or less direct translation of the classical criterion $\operatorname{CJ}(N)$ as defined in Sec. II.A. It circumvents a technical difficulty that has plagued previous attempts at translating the classical criteria C1-C3 into quantum mechanics.[10] The difficulty has been to discriminate between two kinds of commuting operators - those that have a bearing on the question of integrability and those that do not.

Granted that all one-spin systems are quantum integrable as we have asserted in Sec. III, then quantum separability implies quantum integrability. Therefore, any system in which the two degrees of freedom are already separated, is quantum integrable. A two-spin Hamiltonian, for example, which can be expressed as the sum or as the product of two one-spin Hamiltonians,

$$
\widehat{H}_{S}=H_{1}\left(\widehat{\mathbf{S}}_{1}\right)+H_{2}\left(\widehat{\mathbf{S}}_{2}\right), \widehat{H}_{P}=H_{1}\left(\widehat{\mathbf{S}}_{1}\right) H_{2}\left(\widehat{\mathbf{S}}_{2}\right),
$$

can be converted into the form (53) by means of a product unitary transformation $\mathcal{U}_{1}\left(\widehat{\mathbf{S}}_{1}\right) \mathcal{U}_{2}\left(\widehat{\mathbf{S}}_{2}\right)$. Are quantum integrable systems limited to cases that are obviously quantum separable? Do quantum nonintegrable systems exist at all? As a partial and preliminary answer to these questions, we present some numerical evidence for the distinctive spectral consequences of quantum integrability and nonintegrability. This numerical evidence will be inferred, as in Sec. III for one-spin systems, from relations between quantum invariants obtained from time averages of dynamical variables.

\section{B. Integrable two-spin system}

The existence of the special unitary transformation $\mathcal{U}\left(\widehat{\mathbf{S}}_{1}, \widehat{\mathbf{S}}_{2}\right)$ which expresses a given integrable two-spin Hamiltonian as a function of two action operators as in (53) implies that the same is true for any other stationary operator, $I\left(\widehat{\mathbf{S}}_{1}, \widehat{\mathbf{S}}_{2}\right)$ with $[\widehat{I}, \widehat{H}]=0$ :

$$
I\left(\widehat{\mathbf{S}}_{1}, \widehat{\mathbf{S}}_{2}\right)=\mathcal{I}\left(\hat{J}_{1 z}, \hat{J}_{2 z}\right) .
$$

Quantum invariants can be constructed from time averages of an arbitrary dynamical variable (55), and the result must be a function of the two action operators:

$$
\widehat{I}_{F}=\left\langle F\left(\widehat{\mathbf{S}}_{1}, \widehat{\mathbf{S}}_{2}\right)\right\rangle=\left\langle\mathcal{F}\left(\widehat{\mathbf{J}}_{1}, \widehat{\mathbf{J}}_{2}\right)\right\rangle=\mathcal{I}_{F}\left(\hat{J}_{1 z}, \hat{J}_{2 z}\right) .
$$

The last equation in (58) can be established via a systematic process of elimination of the non-stationary components $\widehat{J}_{k x}, \widehat{J}_{k y}, k=1,2$, whose time evolution is known for a given $\mathcal{H}\left(\hat{J}_{1 z}, \hat{J}_{2 z}\right)$. In Sec. III.C we have outlined this process of elimination for a one-spin system.

Our aim here is to show evidence that the expressions $\widehat{H}=\mathcal{H}\left(\hat{J}_{1 z}, \hat{J}_{2 z}\right)$ and $\widehat{I}_{F}=\mathcal{I}_{F}\left(\hat{J}_{1 z}, \hat{J}_{2 z}\right)$ do exist in integrable two-spin systems. An actual calculation of these algebraic expressions via the special unitary transformation $\mathcal{U}\left(\widehat{\mathbf{S}}_{1}, \widehat{\mathbf{S}}_{2}\right)$ is a much more ambitious goal, which is generally out of reach. In classical mechanics our aim is tantamount to showing evidence that the phase flow has an intact torus structure, which implies that the Hamiltonian can be expressed as a function of two action variables $J_{1}, J_{2}$. The more ambitious goal translates into finding the actual functional dependence of $\mathcal{H}$ on $J_{1}, J_{2}$ via the special canonical transformation which eliminates the non-stationary canonical coordinates from the Hamiltonian. That goal is not within reach either, in most applications.

In Sec. III.E the existence of the action operator as a special invariant in generic one-spin systems has been inferred indirectly from the properties of other quantum 
invariants that are either given, such as $\widehat{H}=H(\widehat{\mathbf{S}})$, or can be constructed via time average from non-stationary operators, such as $\widehat{I}_{F}=\langle F(\widehat{\mathbf{S}})\rangle$. The necessary algebraic dependence of any quantum invariant on the action operator $\hat{J}_{z}$ has been corroborated from the manifest algebraic dependence of any two quantum invariants (e.g. $\left.\widehat{H}, \widehat{I}_{F}\right)$ and from the regular pattern of points $\left(E_{n}^{s}\right.$ and $\left.I_{F, n}^{s}\right)$ in any $\Gamma_{s}$ subspace (see Fig. 1).

In a two-spin system, two quantum invariants, $\widehat{I}_{F}=$ $\left\langle F\left(\widehat{\mathbf{S}}_{1}, \widehat{\mathbf{S}}_{2}\right)\right\rangle$ and $\widehat{I}_{G}=\left\langle G\left(\widehat{\mathbf{S}}_{1}, \widehat{\mathbf{S}}_{2}\right)\right\rangle$, may or may not be algebraically dependent, no matter whether the system is quantum integrable or not. If the two invariants happen to be algebraically dependent, then the points $\left(I_{F, \lambda}^{s}, I_{G, \lambda}^{s}\right)$ lie again on a line that is smooth on a scale large compared to the average spacing but, unlike in the one-spin system, the spacings between successive points will be irregular.

If the two-spin system is integrable, QJ(2) implies that any invariant can be expressed as a function of the two quantum actions. Hence, if we pick three quantum invariants, $\widehat{I}_{F}=\mathcal{I}_{F}\left(\hat{J}_{1 z}, \hat{J}_{2 z}\right), \widehat{I}_{G}=\mathcal{I}_{G}\left(\hat{J}_{1 z}, \hat{J}_{2 z}\right)$, $\widehat{I}_{H}=\mathcal{I}_{H}\left(\hat{J}_{1 z}, \hat{J}_{2 z}\right)$, that are pairwise algebraically independent, then the two action operators imply that there exists an algebraic dependence between the three of them. Therefore, the points $\left(I_{F, \lambda}^{s}, I_{G, \lambda}^{s}, I_{H, \lambda}^{s}\right)$ plotted in a 3 -dimensional diagram must lie on a surface that is smooth on a scale large compared to the average spacing and must form a regular pattern reflecting the characteristic spectral properties (54) of the two action operators.

For a demonstration of quantum integrability in a nontrivial application, we consider the two-spin model

$$
H_{\gamma}\left(\widehat{\mathbf{S}}_{1}, \widehat{\mathbf{S}}_{2}\right)=-\left[(1+\gamma) \widehat{S}_{1 x} \widehat{S}_{2 x}+(1-\gamma) \widehat{S}_{1 y} \widehat{S}_{2 y}\right]
$$

with an integrable classical counterpart.[14] The three quantum invariants used here are the energy $\widehat{H}_{\gamma}$ and the stationary operators

$$
\hat{I}_{\mu} \equiv \sqrt{\left\langle\widehat{M}_{\mu}^{2}\right\rangle}, \quad \widehat{M}_{\mu}=\frac{1}{2}\left(\widehat{S}_{1, \mu}+\widehat{S}_{2, \mu}\right), \quad \mu=x, z,
$$

derived from time averages of non-stationary operators.

In Fig. 2(a) we have plotted the eigenvalues $I_{z, \lambda}^{s}$ of the invariant $\widehat{I}_{z}$ versus the energy eigenvalues $E_{\lambda}^{s}$. [16] The open squares and full circles represent all (common) eigenstates with negative energy for spin quantum number $s=35$ and specific transformation properties under the (discrete) symmetry group of $\widehat{H}_{\gamma}$. [13] The arrangement of points $\left(E_{\lambda}^{s}, I_{z, \lambda}^{s}\right)$ reflects the algebraic independence of the two quantum invariants $\widehat{H}_{\gamma}$ and $\widehat{I}_{z}$. On the other hand, if interpreted as the projection of the points $\left(E_{\lambda}^{s}, I_{z, \lambda}^{s}, I_{x, \lambda}^{s}\right)$ onto the $\left(\widehat{H}_{\gamma}, \widehat{I}_{z}\right)$-plane, they illustrate the algebraic dependence of the three quantum invariants $\widehat{H}_{\gamma}, \widehat{I}_{z}, \widehat{I}_{x}$.

Not only are the points $\left(E_{\lambda}^{s}, I_{z, \lambda}^{s}, I_{x, \lambda}^{s}\right)$ located on a surface that is smooth on a large scale compared to the average distance between them, but the spacings between neighboring points on that surface are themselves

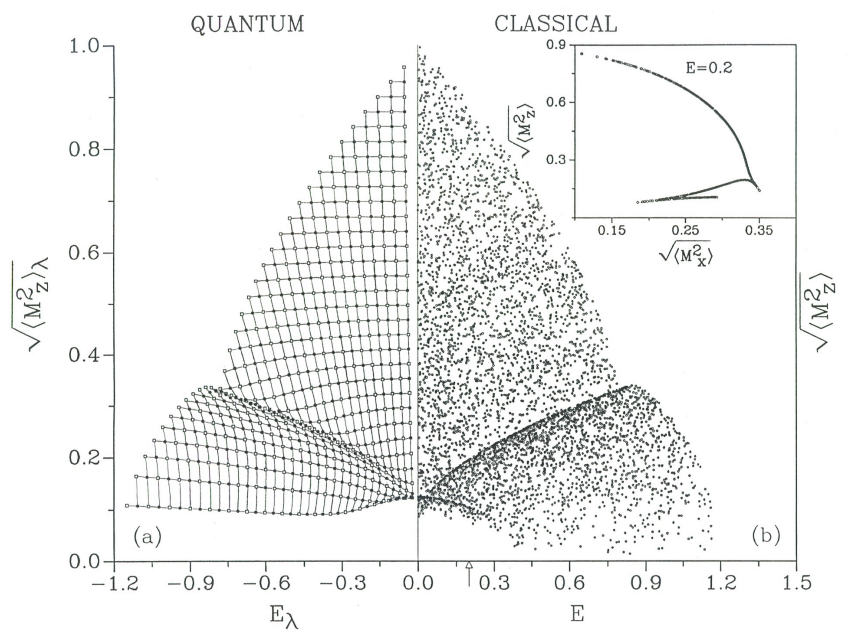

FIG. 2: (a) Quantum invariant $\widehat{I}_{z}=\sqrt{\left\langle\widehat{M}_{z}^{2}\right\rangle}$ versus energy at $E>0$ for two symmetry classes of states (full circles and opens squares) of the integrable quantum two-spin model $\widehat{H}_{\gamma}$ with $\gamma=0.2$ in the subspace $\Gamma_{s}$ with $s=35$ and with $\hbar=1 / \sqrt{s(s+1)}$. (b) Classical invariant-surface $E\left(\sqrt{\left\langle M_{x}^{2}\right\rangle}, \sqrt{\left\langle M_{z}^{2}\right\rangle}\right)$ projected onto the $\left(E, \sqrt{\left\langle M_{z}^{2}\right\rangle}\right)$-plane of the same model in the classical limit $s \rightarrow \infty$. The inset shows invariant $\sqrt{\left\langle M_{z}^{2}\right\rangle}$ versus invariant $\left.\sqrt{\left\langle M_{x}^{2}\right\rangle}\right)$ at energy $E=0.2$. The data points represent time averages over individual trajectories for initial conditions randomly chosen in phase space (main plot) or on the energy hypersurface (inset) (adapted from Ref. 16).

smoothly varying along two linearly independent directions. The regular pattern is compelling evidence for the existence of two natural quantum numbers $\lambda=(m, n)$, reflecting the eigenvalues of two action operators (54) in the subspace $\Gamma_{s=35}$.

Our conclusion, therefore, is that the two-spin system (59) satisfies the quantum integrability condition QJ(2). In Ref. 14 it was proven that the classical counterpart of (59) satisfies the integrability criteria C1-C3, which is equivalent to the condition $\mathrm{CJ}(2)$. However, a constructive proof of CJ(2) for this model may be about as challenging as a a proof of $\mathrm{QJ}(2)$.

The characteristic signatures of quantum integrability in invariants as described in the context of Fig. 2(a) are perfectly in line with those of classical integrability expected in the corresponding classical invariants. The latter are plotted in Fig. 2(b) in exactly the same representation as their quantum counterparts. For a large number of randomly chosen initial conditions $\left(\mathbf{S}_{1}, \mathbf{S}_{2}\right)$ in phase space, we have determined the energy $E$ by insertion into the (stationary) Hamiltonian $H_{\gamma}\left(\mathbf{S}_{1}, \mathbf{S}_{2}\right)$, and the invariants $\sqrt{\left\langle M_{x}^{2}\right\rangle}, \sqrt{\left\langle M_{z}^{2}\right\rangle}$ via time average (9) from the (nonstationary) dynamical variables $M_{\mu}=\left(S_{1, \mu}+S_{2, \mu}\right) / 2$.

For reasons stated in Sec. II.C, classical integrability implies that the points $\left(E, \sqrt{\left\langle M_{x}^{2}\right\rangle}, \sqrt{\left\langle M_{z}^{2}\right\rangle}\right)$ lie on a piecewise smooth invariant-surface. A projection of that 
surface onto the $\left(E, \sqrt{\left\langle M_{z}^{2}\right\rangle}\right)$-plane is shown in Fig. 2(b). The section at $E=0.2$ of the unprojected surface is a piecewise smooth line as shown in the inset. [16]

Each point on the invariant-surface of Fig. 2(b) represents an invariant torus in phase space, specified by two action coordinates. Likewise, each point on the invariant-web of Fig. 2(a) may be said to represent a quantized torus specified by the (discrete) values of two action operators in the given $\Gamma_{s}$ subspace. In the classical system, the smoothness of the invariant-surface guarantees integrability and hence the existence of action coordinates. However, knowledge of classical integrability alone does not provide a general recipe for an analytic solution of the equations of motion. Likewise, the smoothness and regularity of the invariant-web is the hallmark of quantum integrability and hence the existence of action operators, but neither does the knowledge of quantum integrability provide a recipe for an analytic solution of the equation of motion. In classical mechanics, any analytic solution is equivalent to finding the canonical transformation which expresses the Hamiltonian as a function of action coordinates, while in quantum mechanics, any analytic solution is equivalent to finding the unitary transformation which expresses the Hamiltonian as a function of action operators.

\section{Nonintegrable two-spin system}

We have yet to demonstrate that quantum nonintegrability as implied by the condition QJ(2) is associated with distinctive spectral properties. The two-spin Hamiltonian,

$$
\begin{aligned}
H_{\alpha}\left(\widehat{\mathbf{S}}_{1}, \widehat{\mathbf{S}}_{2}\right) & =-\widehat{S}_{1 x} \widehat{S}_{2 x}-\widehat{S}_{1 y} \widehat{S}_{2 y} \\
& -\frac{\alpha}{2}\left[\widehat{S}_{1 x}^{2}-\widehat{S}_{1 y}^{2}+\widehat{S}_{2 x}^{2}-\widehat{S}_{2 y}^{2}\right],
\end{aligned}
$$

has exactly the same symmetries as $H_{\gamma}\left(\widehat{\mathbf{S}}_{1}, \widehat{\mathbf{S}}_{2}\right)$ defined in (59), [13] but its classical counterpart is known to be chaotic for $\alpha \neq 0, \pm 1$. [14] The impact of nonintegrability on both the quantum invariant-web and the classical invariant-surface is conspicuously displayed in Fig. 3. For the classical case we pick three invariants $E, \sqrt{\left\langle M_{x}^{2}\right\rangle}, \sqrt{\left\langle M_{z}^{2}\right\rangle}$ by the same rule as in Sec. IV.B, but now applied to the energy function $H_{\alpha}\left(\mathbf{S}_{1}, \mathbf{S}_{2}\right)$. The lack of a fully intact torus structure in phase space for any nonintegrable model destroys the smoothness of the invariant-surface formed by the points $\left(E, \sqrt{\left\langle M_{x}^{2}\right\rangle}, \sqrt{\left\langle M_{z}^{2}\right\rangle}\right)$ as explained in Sec. II.C.

Figure 3(b) shows the projection of the strange invariant-surface onto the $\left(E, \sqrt{\left\langle M_{z}^{2}\right\rangle}\right)$-plane, as generated from a large number of randomly chosen initial phase points. The section at $E=0.2$ of the unprojected object is plotted in the inset. The pieces (a), (b), (c) of the invariant-surface represent three different types of tori. They have been identified and visualized on a Poincaré map in a previous study. [16] The large interruption separating the fragments marked (a) and (b) is

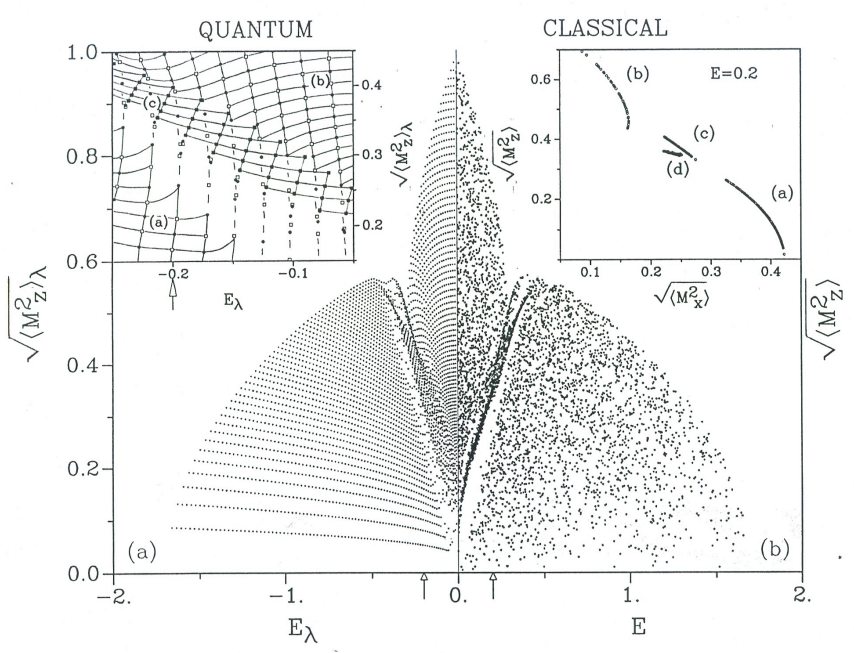

FIG. 3: (a) Quantum invariant $\widehat{I}_{z}=\sqrt{\left\langle\widehat{M}_{z}^{2}\right\rangle}$ versus energy at $E>0$ for the same two symmetry classes of states as in Fig. 2(a) but for the nonintegrable quantum two-spin model $\widehat{H}_{\alpha}$ with $\alpha=0.7$ in the subspace $\Gamma_{s}$ with $s=90$ and with $\hbar=1 / \sqrt{s(s+1)}$. The inset shows the same quantities for states within a window of given size, and for spin quantum number $s=45$. (b) Remnant of the classical invariant-surface $E\left(\sqrt{\left\langle M_{x}^{2}\right\rangle}, \sqrt{\left\langle M_{z}^{2}\right\rangle}\right)$ projected onto the $\left(E, \sqrt{\left\langle M_{z}^{2}\right\rangle}\right)$-plane of the same model in the classical limit $s \rightarrow \infty$. The inset shows invariant $\sqrt{\left\langle M_{z}^{2}\right\rangle}$ versus invariant $\sqrt{\left\langle M_{x}^{2}\right\rangle}$ ) at energy $E=0.2$. The data points represent time averages over individual trajectories for initial conditions randomly chosen in phase space (main plot) or on the energy hypersurface (inset) (adapted from Ref. 16).

due to a band of chaos along a separatrix between regions with an abundance of intact tori. A third major fragment of the invariant-surface, marked (c), has its origin in island chains of tori populating the large chaotic band. For initial conditions within that chaotic region, the points $\left(\sqrt{\left\langle M_{x}^{2}\right\rangle}, \sqrt{\left\langle M_{z}^{2}\right\rangle}\right)$ tend to cluster at $(\mathrm{d})$ in the gap. Strictly speaking, any initial point within that region should yield the same time average. Because of slow convergence, the numerical analysis yields a cluster of points instead. The characteristic pattern is that fragments of the strange invariant-surface are interrupted by gaps, and the gaps are populated by isolated points and smaller fragments of the strange surface. Upon magnification, each fragment reveals its own composition of points and yet smaller fragments ad infinitum.

The signature of quantum nonintegrability in this representation is no less dramatic than that of classical nonintegrability as can be observed in Fig. 3(a). What has been a perfect invariant-web in the integrable case (Fig. $2(\mathrm{a})$ ) now has its fabric torn into pieces. Comparison between the two sides of Fig. 3 reveals a remarkable correspondence between the irregularities in the invariantweb and the fragmentation of the invariant-surface. For example, the two disconnected regions (a) and (b) of the 
intact web in the inset to Fig. 3(a) correspond to two regions with abundant intact tori on opposite sides of a (chaotic) separatrix, and the region (c) of the intact web corresponds to secondary KAM tori within that chaotic band. This correspondence between quantum nonintegrability effects and well-understood phenomena of classical Hamiltonian chaos has been analyzed in greater detail in a previous study. $[13,16]$ All the available evidence for the two-spin model (61) strongly indicates that none of the quantum invariants is a function of two action operators.

Two quantum states which are physically close are also nearby on the invariant-web. When the energies of two such states happen to be nearly degenerate, the consequences depend sensitively on the integrability status of the Hamiltonian. In an integrable model, such as $\widehat{H}_{\gamma}$, the two nearly degenerate states differ in at least one of the two quantum numbers $m, n$ pertaining to the quantum actions (54). Hence the positions of the two states in the web is not at all affected by their near-degeneracy. The fabric stays intact.

In a nonintegrable model, such as $\widehat{H}_{\alpha}$, the natural quantum numbers $m, n$ do no longer exist, and two nearly degenerate states which are also sufficiently close on the web undergo a resonance. The resonance is a form of hybridization, which makes them physically even more alike. The main effects of the resonance as observable in the web are that the two states move closer vertically by a considerable amount and further apart horizontally by a small amount (level repulsion). The fabric is torn as a result of this effect. The degree of near-degeneracy which is needed to trigger an observable resonance is higher in regions of the invariant-web that correspond to regions on the invariant-surface with many intact tori compared to the degree of near-degeneracy needed in those parts of the web that correspond to widespread chaos. This type of resonances and their impact on the level statistics have been investigated more systematically in a recent study of the spin-boson model. [17]

The numerical evidence for the nonintegrability of a classical two-spin system as derived, for example, from a Poincaré map or a strange invariant-surface, is virtually unmistakable if carried out with sufficient circumspection, even though it is not rigorous. Formal proofs of nonintegrability are scarce, essentially limited to systems that are as non-generic as integrable ones are. We maintain that the numerical evidence for quantum nonintegrability which can be inferred from studies of quantum invariants is equally compelling if carried out systematically and with due care. Formal proofs of quantum nonintegrability in the sense of QJ(2), if they can be established at all, will probably be limited to a class of highly non-generic systems as in the classical case.

\section{OUTLOOK}

In this paper we have proposed a quantum integrability condition for $N$-spin systems that promises to be meaningful insofar as it claims to discriminate between two classes of systems whose spectral properties, as exemplified in the invariant-web, differ dramatically. We have shown or cited compelling numerical evidence that none of the two classes is empty. Even though there are strong formal similarities between the quantum integrability condition proposed here and the well-established classical integrability condition, the former does not lean on the latter by way of the correspondence principle. In fact, our strategy has been to avoid a direct translation of the classical integrability criterion in its standard formulation, C1-C3, in order to eliminate the technical difficulties that have haunted previous attempts. The price we have to pay for closing those loopholes is that the new criterion, QJ $(N)$, or its classical counterpart, $\mathrm{CJ}(N)$, is considerably harder to verify in most applications. The evidence strongly suggests that the observed phenomena of quantum chaos are indeed manifestations of quantum nonintegrability, not merely quantum manifestations of classical nonintegrability.

The quantum mechanical action operator $\hat{J}$ was introduced in the early days of quantum mechanics as a natural consequence of Bohr's quantization rule, [18-20] $\oint p d q=n h, n=1,2, \ldots$ Problems with the quantum action do not arise unless we insist on interpreting it as a quantized (stationary) canonical momentum, which calls for a conjugate angle operator. It appears that there is no consensus about how to define such an operator in a satisfactory way.[21-24] However, in our approach the action operator is defined solely on the basis of its spectral properties, and the existence of an angle operator is not required.

However convincing the numerical evidence in support of the meaningfulness and usefulness of the proposed quantum integrability condition may be, it can only serve as a first step in the process of establishing this idea on firm ground. The logical next step, already alluded to in Sec. III.D, would be to test this proposition in the context of a perturbation expansion. A continuation of this study in that direction is currently in progress. The prospects for significant new results may be judged from the following concluding remarks.

In classical mechanics, a generic perturbation of an analytically solved system with one degree of freedom, $\mathcal{H}_{0}(J)$, introduces a dependence on the angle coordinate $\theta$ into the Hamiltonian. The perturbative transformation to new action-angle variables shifts the angle-dependence systematically to higher orders in the expansion parameter. In this process, a series of generating functions for successive canonical transformations is determined. Each generating function contains a factor equal to the inverse of a multiple $n \omega$ of the frequency $\omega=d \mathcal{H}_{0} / d J$ of the unperturbed system. This process is known to converge under some restrictions. 
The situation is drastically different in systems with two degrees of freedom. There exist two fundamental frequencies in the unperturbed system, $\mathcal{H}_{0}\left(J_{1}, J_{2}\right)$, namely $\omega_{1}=\partial \mathcal{H}_{0} / \partial J_{1}, \omega_{2}=\partial \mathcal{H}_{0} / \partial J_{2}$. The basic procedure of the perturbation series remains unchanged: calculate a sequence of generators of infinitesimal canonical transformations which remove the dependence on the angle coordinates $\theta_{1}, \theta_{2}$ from the perturbed Hamiltonian order by order. The integrals required to determine the generators are now found to contain factors equal to the inverse of $n_{1} \omega_{1}+n_{2} \omega_{2}$ with integer coefficients. Any such denominator can take on arbitrarily small values, and thus has the potential for destroying the convergence of the perturbation series. This situation is known in classical mechanics as the problem of small denominators. The KAM theorem provides information about which tori survive a specific perturbation. [25]

Van Vleck's formulation of quantum-mechanical perturbation theory, $[26,27]$ which is akin to the Lie transform method, [28] seems to be suitable for the implementation of the diagonalizing unitary transformation $\widehat{\mathcal{U}}$ for a one-spin system. Here the strategy is to systematically remove the (off-diagonal) creation and annihilation operators (or spin ladder operators) from the Hamiltonian in increasing orders. In this process the generators of infinitesimal unitary transformations contain again factors equal to the inverse of multiples of the frequencies of the unperturbed system.

In quantum systems with two or more degrees of freedom, however, the Van Vleck perturbation expansion may very well be subject to the problem of small de- nominators, possibly in a modified form. In leading order, the generators are indeed found to contain factors equal to the inverse of linear combinations of the unperturbed frequencies with integer coefficients. The associated problems of convergence are known to occur in various perturbational approaches, including transformations to the quantum mechanical Birkhoff-Gustaffson normal form[29], algebraic quantization,[30] and quasidegenerate perturbation theory.[26, 27] From the vantage point of a quantum-mechanical notion of integrability, the lack of convergence of the perturbational schemes now appears in a new light. It may open an avenue to test the criterion $\mathrm{QJ}(N)$ of quantum integrability for a given system on a rigorous basis.

\section{Acknowledgments}

Prior collaborative work with C. Kaufman, E. Magyari, N. Srivastava, H. Thomas, and R. Weber on the dynamics of spin clusters provided much of the initial momentum for this study. We are particularly indebted to N. Srivastava for the production of Figures 2 and 3. The research done in Basel was supported by Schweizerischer Nationalfonds and the research done at URI by the U. S. National Science Foundation, Grant DMR-93-12252. S.W. acknowledges support by Freiwillige Akademische Gesellschaft (Basel). The computations were carried out at the National Center for Supercomputing Applications, University of Illinois at Urbana-Champaign.
[1] Goldstein, M.: Classical Mechanics. New York: AddisonWesley 1950

[2] Lichtenberg, A.J. and Liebermann, M.A.: Regular and Stochastic Motion. New York, Heidelberg, Berlin: Springer-Verlag 1983

[3] Eckhardt, B.: Phys. Rep. 163 (1988) 205

[4] Chaos and Quantum Physics. Giannoni, M. J., Voros, A. and Zinn-Justin, J. (Eds.) Les Houches 1989, NorthHolland 1992

[5] Chaos and Quantum Chaos. Heiss, W. D. (ed.) Lecture Notes in Physics, Vol. 411. Berlin: Springer-Verlag 1992

[6] The Transition to Chaos in Conservative Classical Systems: Quantum Manifestations. Berlin: Springer-Verlag 1992

[7] Hose, G., Taylor, H.S. and Tip, A.: J. Phys. A 17 (1984) 1203

[8] Hietarinta, J.: J. Math. Phys. 25 (1984) 1833

[9] Elyutin, P.V.: Sov. Phys. Usp. 31 (1988) 597

[10] Weigert, St.: Physica D 56 (1992) 107

[11] Ford, J. and Ilg, M.: Phys. Rev A 45 (1992) 6165

[12] Srivastava, N., Kaufman, C., Müller, G., Weber, R. and Thomas, H.: Z. Phys. B 70 (1988) 251

[13] Srivastava, N. and Müller, G.: Z. Phys. B 81 (1990) 137

[14] Magyari, E., Thomas, H., Weber, R., Kaufman, C. and Müller, G.: Z. Phys. B 65 (1987) 363
[15] Perelomov, A.M.: Integrable Systems of Classical Mechanics and Lie Algebras I. Basel: Birkhäuser 1990

[16] Srivastava, N., Kaufman, C. and Müller, G.: Comput. Phys. 4 (1990) 549; 5 (1991) 239; 6 (1992) 84

[17] Cibils, M., Cuche, Y. and Müller, G.: Z. Phys. B (to be published)

[18] Dirac, P.A.M.: Proc. R. Soc. London, 110 (1926) 561

[19] London, F.: Z. Phys 37 (1926) 915

[20] Wentzel, G.: Z. Phys 37 (1926) 80

[21] Newton, R. G.: Ann. Phys. (N.Y.) 124 (1980) 327

[22] Luis, A. and Sánchez-Soto, L.L.: Phys. Rev. A. A (1993) 752

[23] Pegg, D.T. and Barnett, S.M.: Europhys. Lett. 6 (1988) 483

[24] Lévy-Leblond, J.M.: Ann. Phys. (N.Y.) 101 (1976) 319

[25] Thirring, W.: Lehrbuch der Mathematischen Physik, I. New York, Heidelberg, Berlin: Springer-Verlag 1988

[26] Shavitt, I. and Redmon, L.T.: J. Chem. Phys. 73 (1980) 5711

[27] Sibert III, E.L.: J. Chem. Phys. 88 (1988) 4378

[28] Cary, J.R.: Phys. Rep. 79 (1981) 131

[29] Eckhardt, B.: J. Phys. A 19 (1986) 2961

[30] Fried, L.E. and Ezra, G.S.: J. Phys. Chem. 92 (1988) 3144 